\title{
«Das hätte man wissen müssen!» - Der Rückschaufehler und sein Einfluss auf das Fahrlässigkeitsdelikt
}

Roman Elsener *

Der Beitrag behandelt das in der Psychologie seit Langem diskutierte Phänomen, dass das Wissen um den Ausgang eines bestimmten Schadensereignisses die nachträgliche Beurteilung von dessen Voraussehbarkeit signifikant beeinflusst. Der Autor widmet sich der Frage, ob diese Erkenntnisse Einfluss auf unsere Dogmatik der strafrechtlichen Fahrlässigkeitsdelikte haben und falls ja, wie diese zu vermeiden wären. Er kommt zur Einsicht, dass die oft als debiasing-Methode diskutierte Zweiteilung des Prozesses weder über das Tatinterlokut noch über das Erfolgsinterlokut befriedigend gelöst werden kann. Sobald der Strafrichter mit einer Fahrlässigkeitstat befasst ist, weiss er, dass ein Erfolg eingetreten ist, da die folgenlose Fahrlässigkeit nicht strafbar ist. Der Autor schlägt deshalb eine andere Methode zur Vermeidung des Rückschaufehlers vor.

I. Einleitung........................................................................................... 118

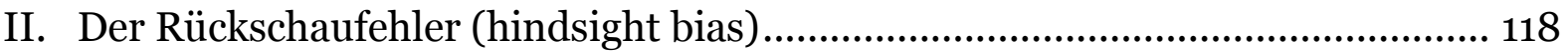

1. Auswirkungen auf die Recht-sprechung ................................................119

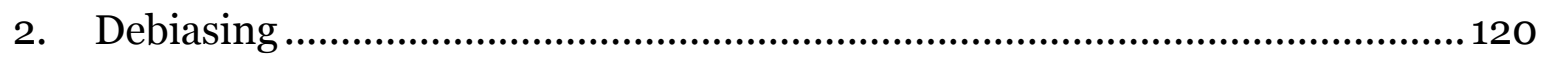

III. Die rückschauende Betrachtung im fahrlässigen Erfolgsdelikt..........................121

1. Die adäquate Kausalität und die Krux der Abstraktion................................. 122

2. Die Sorgfaltspflicht und der trügerische Gefahrensatz .............................. 124

3. Die Voraussehbarkeit als eigenständiges Tatbestandsmerkmal ................... 125

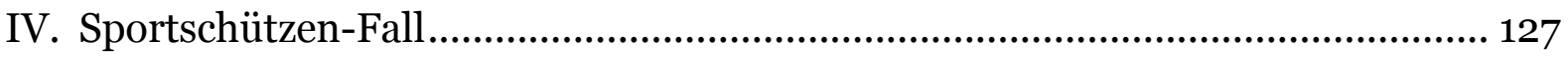

V. Methoden zur Verminderung des Rückschaufehlers im Strafrecht ...................128

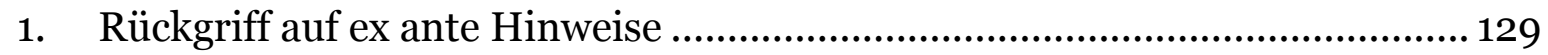

2. Beweiswürdigung im Strafprozess ........................................................... 130

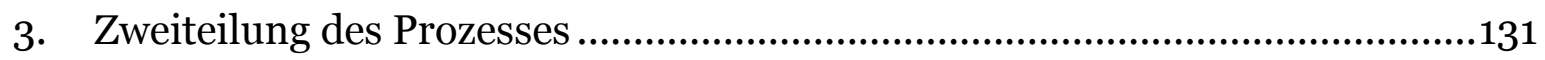

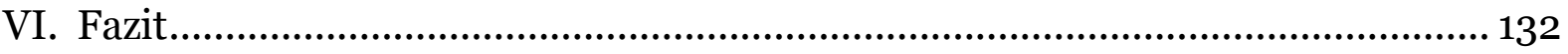

Zitiervorschlag: Roman Elsener, «Das hätte man wissen müssen!» - Der Rückschaufehler und sein Einfluss auf das Fahrlässigkeitsdelikt, in: sui-generis 2015, S. 117

URL: $\quad$ sui-generis.ch/16

* Roman Elsener, MLaw. Geboren 1986; Studium der Rechtswissenschaft an der Universität Luzern. Dieser Beitrag ist eine Kurzfassung der Masterarbeit vom Dezember 2014.

Dieses Werk ist lizenziert unter einer Creative Commons Namensnennung - Weitergabe unter gleichen Bedingungen 4.0 International Lizenz. 


\section{Einleitung}

1 «Das hätte man wissen müssen!» So oder ähnlich reagieren wir oft auf Unfälle, bei denen jemand einen Fehler mit voraussehbaren Folgen begangen hat. Doch waren diese Folgen tatsächlich voraussehbar? Im Rückblick wird die Vorhersehbarkeit nämlich oftmals überschätzt. «Hinterher ist man immer schlauer», lautet denn auch eine bekannte Redewendung.

Was früher eine blosse Volksweisheit war, wird seit ca. vier Jahrzenten vermehrt wissenschaftlich untersucht. Nebst der Psychologie, der Politikwissenschaft sowie der Ökonomie setzt sich vermehrt auch die Rechtswissenschaft mit dem Phänomen des sogenannten Rückschaufehlers (hindsight bias) auseinander ${ }^{1}-$ und das mit gutem Grund: Eine gerichtliche Beurteilung erfolgt zwangsweise immer erst nachdem etwas passiert ist (ex post). Trotzdem dürfen die Richter für ihr Urteil oft nur das Wissen und die Umstände berücksichtigen, die auch tatsächlich im Tatzeitpunkt bekannt gewesen sind (ex ante). Dieser Spagat zwischen dem Wissen ex post und der Beurteilung ex ante ist insbesondere im Strafrecht von grosser Bedeutung. Das Strafrecht soll durch Verbote und Gebote menschliches Verhalten lenken. Gelenkt werden kann nur das Verhalten eines Menschen, nicht aber der Zufall. Folgerichtig darf auch bloss das Verhalten an sich bestraft werden und nicht der zufällige Erfolg. Nur so kann der präventive Zweck des Strafrechts überhaupt seine

\footnotetext{
Mark Schweizer, Kognitive Täuschungen vor Gericht, Eine empirische Studie, Diss. Zürich 2005, 33; Vito Roberto/Kristoffel Grechenig, Rückschaufehler (hindsight bias) bei Sorgfaltspflichtverletzungen, in: ZSR 2011, 5, $6 \mathrm{f}$.
}

Wirkung erzielen.

3 Ein Richter steht somit vor der schwierigen Herausforderung, sich in den Beschuldigten und die Umstände im Tatzeitpunkt hinein zu versetzen. Alleine das Verhalten eines Beschuldigten soll über die strafrechtliche Schuld entscheiden. Der Richter darf sich dabei nicht durch die Erfahrungen der Rückschau beeinflussen lassen, denn wie gesagt: «Hinterher ist man immer schlauer.»

4 Nachfolgend wird der Rückschaufehler kurz umrissen und sein Einfluss auf die Rechtsprechung untersucht (II). Anschliessend wird das fahrlässige Erfolgsdelikt auf die Anfälligkeit einer Rückschauverzerrung hin überprüft (III). Die Anfälligkeit wird anhand des Sportschützen-Falles kurz aufgezeigt (IV). In einem weiteren Schritt werden mögliche Debiasing-Methoden angeführt und deren Tauglichkeit besprochen (V).

\section{Der Rückschaufehler (hindsight bias)}

5 In der kognitiven Psychologie ist der Rückschaufehler inzwischen einer der meist untersuchten kognitiven Fehler. ${ }^{2}$ Die ersten empirischen Studien zum Rückschaufehler wurden in der psychologischen Forschung in den siebziger Jahren durchgeführt. Seit den neunziger Jahren hat die Literaturdichte dazu stark zugenommen. 3

2 Jeffrey J. Rachlinski, Heuristics and Biases in the Courts: Ignorance or Adaption?, in: Oregon Law Review 79/2000, 61, 66.

3 Schweizer (Fn. 1), 33; Ulrich Falk, Urteilsverzerrungen: hindsight bias und anchoring. Einleitende Fragen zu einem interdisziplinären Problem, in: Vincere scis, victoria uti nescis: Aspekte der Rückschauverzerrung in der alten Geschichte, hrsg. 
6 Die wohl bekannteste Studie zum Rückschaufehler und der definitive Nachweis des Phänomens gelang Baruch Fischhoff 1975. Er nannte seine Hypothese «creeping determinism» (schleichender Determinismus) und bewies damit, dass das Wissen über den Ausgang eines Ereignisses die eigene Wahrnehmung bezüglich der Wahrscheinlichkeit dieses Ausgangs signifikant beeinflusst.4 Dazu werden die bekannten Indikatoren, die eher für den eingetretenen Ausgang sprechen, viel höher gewichtet, als jene, die für einen anderen Ausgang sprechen. 5

\section{Auswirkungen auf die Recht- sprechung}

7 Wie sich der Rückschaufehler konkret auf die Rechtsanwendung auswirken kann, zeigt ein Experiment von Kamin/Rachlinski. ${ }^{6}$ Das Augenmerk war dabei auf die unabdingbare Gegebenheit gerichtet, dass ein Richter ex post über eine ex ante Sachlage urteilen muss. Die Autoren simulierten für die ex ante Gruppe (foresight) auf Tonband eine Beratungssitzung eines städtischen Planungsausschusses. Der Planungsausschuss musste entscheiden, ob bei einer neu gebauten Zugbrücke ein Brücken-

von Kay Brodersen, Berlin 2008, 9 ,

13; Roberto/Grechenig (Fn. 1), 6.

4 Falk (Fn. 3), 14; Baruch Fischhoff, Hindsight $\neq$ foresight: The effect of outcome knowledge on judgment under uncertainty, in: Journal of Experimental Psychology: Human Perception and Performance 1/1975, 288, $288 \mathrm{ff}$.

5 Fischhoff (Fn. 4), 291 f.; LaBine/LaBine, Determinations of Negligence and the Hindsight Bias, in: Law and Human Behavior 20/1996, 501, 503; David A. Schkade/Lynda M. Kilbourne, Expectation Outcome Consistency and Hindsight Bias, in: Organizational Behavior and Human Performance 49/1991, 105, 107.

$6 \quad$ Kim A. Kamin/Jeffrey J. Rachlinski, Ex Post $\neq$ Ex Ante: Determining Liability in Hindsight, in: Law and Human Behavior 19/1995, 89, 89 ff. wärter eingestellt werden sollte oder nicht. Anlass dafür war die von Experten begründete Gefahr, dass durch die neue Brücke bei Eis oder Geröll ein Damm entstehen und zu Hochwasser in besiedeltem Gebiet führen könnte. Diese Gefahr könnte mit einem Brückenwärter verringert werden. Die Kosten für einen solchen Brückenwärter wären jedoch ziemlich hoch, berücksichtigte man die geringe Wahrscheinlichkeit einer Überflutung. Mit denselben Informationen und Aussagen aus der Beratungssitzung wurde ein Gerichtsprozess für die ex post Gruppe (hindsight) simuliert. In diesem Gerichtsprozess ging man davon aus, dass der Planungsausschuss seinerzeit keinen Brückenwärter eingestellt hatte und es zu einer Überflutung mit Sachschäden an Privateigentum kam.7

8 Bei der ersten Gruppe (foresight) stimmten $24 \%$ der Probanden für die Einstellung eines Brückenwärters. Das bedeutet, dass drei Viertel die Wahrscheinlichkeit einer Überflutung anhand der zur Verfügung stehenden Informationen als so gering erachteten, dass kein Brückenwärter eingestellt werden musste. In der zweiten Gruppe (hindsight), die nun mit dem Wissen um die Flutkatastrophe rückschauend beurteilen musste, ob mit den damaligen Informationen ein Brückenwärter hätte eingestellt werden müssen, stimmten 56.9 \% für einen Brückenwärter. 8 Die Beurteilung der Probanden wurde durch das nachträgliche Wissen deutlich verändert. Die Resultate von Kamin/Rachlinski veranschaulichen, wie die richterliche Beurteilung durch den Rückschaufehler verfälscht werden kann. Die durch das Wissen des Ausgangs

\footnotetext{
Kamin/Rachlinski (Fn. 6), 94 ff.

8 Kamin/Rachlinski (Fn. 6), 98 f.
} 
überschätzte Voraussehbarkeit eines Ereignisses kann die Entscheidung des Richters nachweislich beeinflussen. Im Recht ist das Tatbestandsmerkmal der Voraussehbarkeit, das besonders anfällig auf Rückschaufehler ist, allgegenwärtig. 9 Die bis heute veröffentlichten Studien, die rechtliche Sachverhalte untersuchen, beschränken sich jedoch meist auf das zivilrechtliche Deliktrecht. Dennoch ist im Strafrecht, das für einen Menschen die einschneidendsten Massnahmen bereitstellt, die Bedeutung des Rückschaufehlers mindestens ebenso gross, wenn nicht noch grösser. ${ }^{10}$

\section{Debiasing}

9 Die Ursache des Rückschaufehlers wird heute in der fehlerhaften kognitiven Wahrnehmung gesehen. In der Vorschau wird anhand der zur Verfügung stehenden Informationen unbewusst eine plausible Geschichte entwickelt, in welcher auch die Wahrscheinlichkeit der möglichen Folgen abgeschätzt wird. Durch das spätere Bekanntwerden der tatsächlich eingetretenen Folge werden dann die Indikatoren, die für die nun bekannte Folge sprechen, plötzlich höher gewertet als zuvor. Mit dem neuen Wissen wird die früher entwickelte Geschichte verworfen und neu geschrieben. Die tatsächliche Voraussehbarkeit wird so im Nachhinein überschätzt und damalige Entscheidungsträger werden für ihre fehlende Voraussicht kritisiert. ${ }^{11}$

\footnotetext{
Kamin/Rachlinski (Fn. 6), 102.

1o Falk (Fn. 3), 9.

11 Jay J. Christensen-Szalanski/Cynthia F. Willham, The Hindsight Bias: A Meta analysis, in: Organizational Behavior and Human Decision Processes 48/1991, 147, 163 f.; Fischhoff (Fn. 4), 291 f.; Pamela D. Pengelley, Foresight in Hindsight: An Insight into Ahmed v. Stefaniu and Debiasing Legal Evaluations of Reasonable Care, in: Annual Review
}

10 Nach heutigem Wissensstand ist es kaum möglich, den Rückschaufehler durch blosse Aufklärung oder Warnung zu verhindern. Versuchspersonen wurden ausdrücklich auf den Rückschaufehler hingewiesen und sollten diesen dadurch nicht mehr begehen. ${ }^{12}$ Die Ergebnisse mehrerer Studien zeigen jedoch, dass alleine durch das Bewusstsein um den Effekt keine nachweisbare Verminderung erzielt werden kann. ${ }^{13}$

11 Das bisher einzige erfolgversprechende Debiasing wurde dadurch erreicht, dass sich die Probanden bewusst mit den alternativen Ausgangsmöglichkeiten auseinandersetzen mussten. Probanden wurden in Experimenten aufgefordert, zu jedem möglichen Ausgang je ein Argument niederzuschreiben. ${ }^{14}$ Diese Methode kann die kognitive Täuschung zwar nicht gänzlich eliminieren aber deutlich minimieren, wie mehrere Studien bestätigen. ${ }^{15}$ Diese Debiasing-Methode setzt

of Civil Litigation 2007, 49, 57 f.; Jeffrey J. Rachlinski, A Positive Psychological Theory of Judging in Hindsight, in: The University of Chicago Law Review 65/1998, 571, 584 ff.; Rachlinski (Fn. 2), 68 f.; Roberto/Grechenig (Fn. 1), 17; Schkade/Kilbourne, 107 f.; Alison C. Smith/Edith Greene, Conduct and it's Consequences: Attempts at Debiasing Jury Judgments, in: Law and Human Behavior 29/2005, 505, 524; Merrie Jo Stallard/ Debra L. Worthington, Reducing the Hindsight Bias Utilizing Attorney Closing Arguments, in: Law and Human Behavior 22/1998, 671, 672.

12 Fischhoff (Fn. 4), 296.

${ }_{13}$ Hal R. Arkes et al., Eliminating the Hindsight Bias, in: Journal of Applied Psychology 73/1988, 305, 305; Fischhoff (Fn. 4), 294 f.;Chris Guthrie/Jeffrey J. Rachlinski/Andrew J. Wistrich, Inside the Judicial Mind, in: Cornell Law Review 86/2001, 777, 825; Kim A. Kamin/Jeffrey J. Rachlinski, Ex Post $\neq$ Ex Ante: Determining Liability in Hindsight, in: Law and Human Behavior 19/1995, 89, 98; Rachlinski (Fn. 11), 70; Smith/Greene (Fn. 11), 524; a. M. Stallard/Worthington (Fn. 11), $680 \mathrm{ff}$.

14 Arkes et al. (Fn. 13), 305.

15 Kamin/Rachlinski (Fn. 6), 92; Rachlinski (Fn. 11), 
jedoch voraus, dass überhaupt eine beschränkte Anzahl von Möglichkeiten vorgelegt werden kann. Das ist einfach umzusetzen in selbst erstellten Experimenten. In einem Strafprozess besteht diese Ausgangslage jedoch nicht immer. Ein realer Sachverhalt ist oft zu komplex, um einem Verhalten einfach eine beschränkte Anzahl Ausgangsmöglichkeiten zuzuschreiben. Zudem wurde festgestellt, dass bei der Aufforderung, mehrere Argumente niederzuschreiben, der Debiasing-Effekt auch aufgehoben werden kann. ${ }^{16}$ Ein Teil der Lehre spricht in solchen Fällen gar von einem BackfireEffekt: Muss der Beurteilende zu viele Argumente abrufen, kann dies den Rückschaufehler sogar verstärken. ${ }^{17}$ Zusammenfassend kann gesagt werden: Es gibt bis heute keine Methode, den Rückschaufehler vollständig zu eliminieren. Er hat sich als einer der widerstandsfähigsten kognitiven Fehler erwiesen. ${ }^{18}$

\section{Die rückschauende Betrachtung im fahrlässigen Erfolgsdelikt}

Im Folgenden wird überprüft, ob die Tatbestandsmerkmale des fahrlässigen Erfolgsdelikts anfällig sind auf Rückschaufehler. Für die Überprüfung der

587; Rachlinski (Fn. 2), 71; Lawrence J. Sannal Norbert Schwarz/Eulena M. Small, Accessibility experiences and the hindsight bias: I knew it all along versus it could never have happened, in: Memory \& Cognition 30/2002, 1288, $1288 \mathrm{f}$.

16 Martin F. Davies, Reduction of hindsight bias by restoration of foresight perspective: Effectiveness of foresight encoding and hindsight retrieval strategies, in: Organizational Behavior and Human Decision Processes 40/1987,

50, 64; Sanna/Schwarz/Small (Fn. 15), 1289.

17 Pengelley (Fn. 11), 69; Sanna/Schwarz/Small (Fn. 15), $1289 \mathrm{ff}$.

18 Guthrie/Rachlinski/Wistrich (Fn.

13), 801; Rachlinski (Fn. 2),

70; Roberto/Grechenig (Fn. 1), 20. einzelnen Tatbestandsmerkmale muss man sich vorgängig überlegen, ob die Beurteilung des Richters nachträglich erlangte Tatsachen (ex post) oder eben gerade nur das Wissen des Beschuldigten zum Tatzeitpunkt (ex ante) berücksichtigen darf. Der Rückschaufehler kann nur dort in Erscheinung treten, wo auch eine Rückschau stattfindet. Umgekehrt erübrigt sich eine nähere Prüfung bei Tatbestandsmerkmalen, deren Beurteilung nicht in der Rückschau stattfinden und später erlangtes Wissen berücksichtigen dürfen.

Zieht zum Beispiel die Verletzung eines Opfers eine dauerhafte Arbeitsunfähigkeit nach sich und ist damit eine schwere Körperverletzung gegeben? Oder handelt es sich lediglich um einen leicht verheilenden Knochenbruch und somit um eine leichte Körperverletzung? Für diese Fragen kann ein Richter auf Wissen zurückgreifen, dass erst nach der Tat bekannt wurde. Ob ein Erfolg eingetreten ist und wenn ja auf welche Weise wird nach dem Ereignis festgestellt. Oder anders formuliert, die Beurteilung erfolgt ex post und darf Ex-post-Wissen berücksichtigen. Der Rückschaufehler hat somit bei der Subsumtion des Taterfolgs keinerlei Bedeutung. Ebenfalls Ex-post-Wissen berücksichtigt werden darf bei der Beurteilung der natürlichen Kausalität zwischen der Tathandlung und dem eingetretenen Erfolg. Die Conditio-sine-qua-nonFormel berücksichtigt naturwissenschaftlich nachgewiesene Erkenntnisse und nachträglich bekannte Tatsachen. ${ }^{19}$

19 BGE 135 IV 56 E. 2.2 S. 65; Andreas Donatsch, Sorgfaltsbemessung und Erfolg beim Fahrlässigkeitsdelikt, Habil. Zürich 1987, 264;Andreas Donatsch/Brigitte Tag, Strafrecht I, Verbrechenslehre, 9. Aufl., Zürich 2013, 339; Mirjam Annika Frei, Der rechtlich relevante Kausalzusammenhang 
Bei den genannten Tatbestandsmerkmalen erübrigt sich eine weitere Überprüfung. Es findet schlicht keine Rückschau statt.

14 Anders verhält es sich bei der Beurteilung der adäquaten Kausalität (1), der relevanten Sorgfaltspflicht (2) und der Voraussehbarkeit eines Taterfolgs (3). Die Beurteilung des Richters erfolgt zwar auch nach der Tat (ex post), er darf aber nur das Wissen des Beschuldigten zum Tatzeitpunkt berücksichtigen (ex ante). Die genannten Tatbestandsmerkmale sind in der Rückschau zu beurteilen, sodass die Gefahr eines Rückschaufehlers besteht. Nachfolgend werden daher diese Tatbestandsmerkmale im Fokus stehen. Dass zum Beispiel die Entscheidung keinen Brückenwärter einzustellen zu einer Überflutung geführt hat ist im Nachhinein sonnenklar. Ob der Beschuldigte oder ein Dritter dies zum Zeitpunkt des Entscheids voraussehen konnte ist aber eine völlig andere Frage. Das nachträgliche Wissen, dass es tatsächlich zu einer Überflutung gekommen ist, darf in diesem Fall keine Rolle spielen.

\section{Die adäquate Kausalität und die Krux der Abstraktion}

Nicht jede natürlich kausale Verursachung eines Erfolgs ist auch strafrechtlich relevant. Dies berücksichtigt das Bundesgericht mit der zusätzlichen Prüfung der adäquaten Kausalität. Ein Verhalten ist nur dann strafbar, wenn es nach dem gewöhnlichen Lauf der Dinge

im Strafrecht im Vergleich mit dem Zivilrecht, Diss. Zürich 2010, 31; Philippe Graven/Bernhard Sträuli, L'infraction pénale punissable, 2. Aufl., Bern 1995, 90; Günter Stratenwerth, Schweizerisches Strafrecht, Allgemeiner Teil I: Die Straftat, 4. Aufl., Bern 2011, § 9 N 41. und den Erfahrungen des Lebens geeignet war, einen Erfolg in der Art des eingetretenen herbeizuführen oder mindestens zu begünstigen. ${ }^{20}$

16 Im Gegensatz zur natürlichen Kausalität erfolgt die Prüfung der adäquaten Kausalität mit dem Wissen ex ante. ${ }^{21}$ Entscheidend ist die allgemeine Lebenserfahrung zum Zeitpunkt der Handlung. Die durch die Rückschau erlangten Erkenntnisse dürfen dabei keine Rolle spielen. Den Ausgangspunkt soll nicht der eingetretene Erfolg bilden, sondern die erfahrungsgemässe Möglichkeit, einen solchen zu bewirken. ${ }^{22}$ Es macht nur dann Sinn, ein Verhalten zu bestrafen, wenn man im Zeitpunkt dieses Verhaltens die mögliche Verletzung erkennen konnte. Andernfalls wäre dies die Rückkehr zum Erfolgsstrafrecht. ${ }^{23}$ Damit die Erkennbarkeit des Erfolgs im Zeitpunkt der Tat bestimmt werden kann, ist es von grossem Nutzen für den Richter, wenn er auf bestehende Hinweise, z. B. in Form von Standards, zurückgreifen kann. Gibt es beispielsweise für den Arzt ein klares Protokoll, wie er bei einer Anamnese vorzugehen hat,

20 BGE 135 IV 56 E. 2.2 S. 65; Donatsch (Fn. 19), 259 f.; Martin Killias et al., Grundriss des Allgemeinen Teils des Schweizerischen Strafgesetzbuchs, Bern 2009, 406; Marcel Alexander Niggli/Stefan Maeder, in: Basler Kommentar zum Schweizerischen Strafgesetzbuch I, hrsg. von Marcel Alexander Niggli/Hans Wiprächtiger, Art. 1 110, 3. Aufl., Basel 2013, Art. 12

N 94; Stratenwerth(Fn. 19), § 16 N 6; Stefan Trechsel/Peter Noll, Schweizerisches Strafrecht, Allgemeiner Teil I: Allgemeine Voraussetzungen der Strafbarkeit, 6. Aufl., Zürich 2004, 269 f.

21 BGE 135 IV 56 E. 2.2 S. 65; Frei (Fn. 19), 94; Graven/Sträuli (Fn. 19), 91; Stratenwerth (Fn. 19), § 9 N 25; a. M. Donatsch (Fn. 19), 262 f.; im Zivilrecht ex post: Frei (Fn. 19), 611.

22 Donatsch (Fn. 19), 261; Frei (Fn. 19), 94.

23 Donatsch (Fn. 19), 101; BSK-StGB I-Niggli/Mae$\operatorname{der}$ (Fn. 20), Art. 12 N 16 ff., 94;Stratenwerth (Fn. 19), § 9 N 24. 
kann er nachträglich nicht geltend machen, er hätte die innere Blutung nicht erkannt, wenn dies auf die Nichtbeachtung dieses Protokolls zurückzuführen ist. ${ }^{24}$ Fehlen hingegen solche vergleichenden Standards, besteht die grosse Gefahr, durch den Rückschaufehler fast alles als erkennbar anzusehen. 25

Unklarheit bei der Anwendung der Adäquanztheorie herrscht in der Frage, wie hoch der Abstraktionsgrad sein darf. Je nachdem, wie fest der Geschehensablauf verallgemeinert wird, kann dies zu unterschiedlichen Ergebnissen führen. ${ }^{26} \mathrm{Ab}$ einer gewissen Abstraktion ist jedes Verhalten adäquat kausal für den Erfolg. Veranschaulicht wird dies etwa im sogenannten Motormäher-Fall. ${ }^{27}$ Dabei kollidierte ein Lastwagenführer, der mit 40 $\mathrm{km} / \mathrm{h}$ unterwegs war, mit einem korrekt fahrenden Stationswagen. Durch die Wucht des Zusammenpralles wurde der auf der kleinen Ladefläche des Stationswagens mitgeführte $250 \mathrm{~kg}$ schwere Motormäher, der lediglich durch Einschaltung des Rückwärtsganges und ein vom rechten Handgriff zur hintern Stossstange lose gespanntes Seil gesichert war, stark nach vorne geworfen. Ein Eisennocken des Motormähers durchbohrte daraufhin den Führersitz und fügte dem Führer des Stationswagens tödliche Verletzungen im Rücken zu. Die Wucht des Aufpralls an sich führte beim Stationswagenfahrer gerade nicht zu tödlichen Verletzungen. Alleine die Verletzungen

\footnotetext{
4 Vgl. BGE 130 IV 7 E. 4.3 S. 14.

5 Schweizer (Fn. 1), 677.

26 Frei (Fn. 19), 82 ff.; BSK-StGB I-Niggli/Maeder (Fn. 20), Art. 12 N 95; Stratenwerth (Fn. 19), § 9 N 26.

27 Frei (Fn. 19), 82 ff.; BSK-StGB I-Niggli/Maeder (Fn. 20), Art. 12 N 95; Stratenwerth (Fn. 19), § 9 N 26.
}

durch den Motormäher führten zu dessen Tod. Das Bundesgericht schreibt dies auch genauso in seinen Erwägungen, wendet danach aber einen zu hohen Abstraktionsgrad bei der Adäquanzformel an, indem es lediglich nach der Möglichkeit fragt, ob «bei einem Zusammenstoss in der unübersichtlichen Einmündung Menschen tödlich verletzt werden konnten». .28

18 Die Unklarheiten in der Adäquanzprüfung bezüglich Objektivität und Abstraktion sind problematisch. Durch den grossen Spielraum in der Frage, welches Wissen einem neutralen Beobachter zugemutet und wie abstrakt der Sachverhalt betrachtet werden soll, wird dem Rückschaufehler eine nährende Grundlage gegeben. Für den Richter besteht ein grösserer Ermessensspielraum und seiner intuitiven Meinung wird mehr Gewicht gegeben als der eigentlichen objektiven Ansicht. ${ }^{29}$ Die Auswirkung wird im erwähnten Motormäher-Fall deutlich: Die Adäquanz wird im Entscheid so weit ausgedehnt, dass das Selbstverschulden des Stationswagenführers untergeht respektive dem Beschuldigten angelastet wird. Auch in zahlreichen weiteren Entscheiden wurde die Adäquanz bejaht, indem das Gericht einen sehr hohen Abstraktionsgrad anwendete oder ungewöhnlich hohe Anforderungen an den Beschuldigtenstellte. $3^{30}$

28 BGE 86 IV 153 E. 2.

29 Guthrie/Rachlinski/Wistrich (Fn. 13), $825 \mathrm{ff}$.

30 BGE 131 IV 145 (übersetzt in: Pra 95/2006, Nr. 70, S. 496 ff.); 127 IV 62; 121 IV 286; 101 IV 67; 100 IV 279; 92 IV 86; 88 IV 107; Urteil des Bundesgerichts 6B_543/2012 vom 11. April 2013; 6B_611/2011 vom 31. Januar 2012; 6B_734/2007 vom 25. Februar 2008; 6S_155/2003 vom 19. August 2003; 6S_529/2001 vom 18. Februar 2002. 
19 Die Anwendung der adäquaten Kausalität wird in der Lehre entsprechend kritisiert. ${ }^{1}$ Es gibt bisher nur sehr wenige Fälle, in denen die adäquate Kausalität verneint wurde. $3^{2}$ Leider lässt sich - ironischerweise - im Nachhinein kaum feststellen, inwiefern der Rückschaufehler für diese rigide Praxis des Bundesgerichts verantwortlich ist. Es zeigt sich jedoch in mehreren Entscheiden, dass die Anwendung der adäquaten Kausalität immer mehr einer strikten Zurechnung gleichkommt.33 Dies ist denn auch eine der in der Wissenschaft befürchteten Folgen, die aufgrund des Rückschaufehlers eintreten können. 34

Abschliessend bleibt festzuhalten, dass zwar der Zusammenhang zwischen dem Rückschaufehler und der hohen Abstraktion bei der Adäquanzprüfung nicht bewiesen werden kann. Es besteht aber die Gefahr, durch eine grenzenlose Abstraktion eher dem Rückschaufehler zu unterliegen. In der deutschen Rechtswissenschaft vermuten daher einige Autoren bereits einen Zusammenhang zwischen der Ausuferung der Adäquanz und dem Rückschaufehler.35

${ }_{31}$ Gunther Arzt, $50 \mathrm{~km} / \mathrm{h}$ innerorts - zu schnell?, Dogmatische Bemerkungen zu BGE 121 IV 286, in: SJZ 92/1996, 305, 305 f.; Frei(Fn. 19), 118 ff., 612 ff.; Killias et al. (Fn. 20), 407; BSK-StGB INiggli/Maeder (Fn. 20), Art. 12

N 95; Trechsel/Noll (Fn. 20), 270.

32 BGE 115 IV 100 E. 2 S. 103; Urteil des Bundesgerichts 6S_262/2006 vom 23. Oktober 2006 E. 2.2; BSK-StGB I-Niggli/Maeder (Fn. 20), Art. 12 N 95; Schweizer (Fn. 1), 676.

33 Vgl. die Nachweise in Fn. 30.

34 Rachlinski (Fn. 11), 596 ff.; Roberto/Grechenig (Fn. 1), 11.

35 Falk (Fn. 3), 10 ff.; Ulrich Sommer, Effektive Strafverteidigung: ein Handbuch für die Theorie und Praxis der Strafverteidigung - mit grundlegenden Erläuterungen zu Recht, Psychologie und zu den Überzeugungstechniken des Strafverteidigers, Köln 2011, 171 f.

\section{Die Sorgfaltspflicht und der trüge- rische Gefahrensatz}

21 Für die Beurteilung, welches Verhalten nach den Tatumständen angebracht ist, das heisst welche Sorgfalt in der konkreten Situation aufzubringen ist, werden Sorgfaltspflichten aus dem Gesetz sowie aus nichtstaatlichen Vorschriften, Reglementen oder Merkblättern herangezogen. ${ }^{6}$ Das Bundesgericht betont jedoch explizit, dass umgekehrt die Einhaltung oder das Nichtvorhandensein einer solchen Vorschrift nicht automatisch einen Freispruch nach sich zieht. Vielmehr kann sich der Vorwurf der Sorgfaltspflichtverletzung nach Lehre und Rechtsprechung auch auf den allgemeinen Gefahrensatz stützen. 37 Welche Gefahr diese «Generalklausel» birgt, zeigt sich mit Blick auf die Rückschauproblematik.

22 Durch das Heranziehen einer bestehenden Sorgfaltsnorm kann eine konkrete Sorgfaltspflicht ermittelt werden. Besteht eine solche Sorgfaltsnorm bereits vor dem Zeitpunkt der Beurteilung (ex ante), ergibt sich daraus eine objektive Grundlage. Was dies konkret bedeutet verdeutlicht beispielsweise Art. 46 Abs. 2 Bauarbeitenverordnung des Bundes (BauAV)38: Das Bundesgericht hat in einem

36 BGE 140 II 7 E. 3.4 S. 10; 130 IV 7 E. 3.3 S. 11; Urteil des Bundesgerichts 6B_126/2014 vom 13. Mai 2014 E. 1.1; 6B_543/2012 vom 11. April 2013 E. 1.3.2; Donatsch/Tag (Fn. 19), 347 ff.; BSKStGB I-Niggli/Maeder (Fn. 20), Art. 12 N 111; Kurt Seelmann, Strafrecht Allgemeiner Teil, 5. Aufl., Basel 2012, 168; Trechsel/Noll (Fn. 20), 270 ff.

37 BGE 140 II 7 E. 3.4 S. 10; 127 IV 62 E. 2.d S. 65; Donatsch/Tag (Fn. 19), 351; BSK-StGB I-Niggli/ Maeder (Fn. 20), Art. 12 N 112;Seelmann (Fn. 36), 168; Trechsel/Noll (Fn. 20), 273.

38 Verordnung über die Sicherheit und den Gesundheitsschutz der Arbeitnehmerinnen und Arbeitnehmer bei Bauarbeiten, SR 832.311.141; Art. 46 Abs. 2 BauAV lautet: «Der Abstand des Belages von der Fassade darf in keiner Bauphase $30 \mathrm{~cm}$ übersteigen. 
seiner Entscheide diese Norm für die Beurteilung der geforderten Sorgfaltspflicht herangezogen. Es verurteilte den Bauleiter, weil aufgrund eines zu grossen $\mathrm{Ab}$ standes zwischen dem Gerüstbelag und der Fassade (nämlich $43 \mathrm{~cm}$ anstatt der gesetzlich geforderten $30 \mathrm{~cm}$ ) ein Arbeiter vom Gerüst stürzte. 39

Durch derartige Sorgfaltsnormen lässt sich relativ einfach ermitteln, welche Risiken der Beschuldigte im Tatzeitpunkt eingehen durfte. Dadurch entfällt die problematische rückschauende Beurteilung der geforderten Sorgfalt. Der Rückschaufehler kann so durch bestehende Normen stark vermindert werden. ${ }^{40}$ In der heutigen komplexen Welt ist es jedoch kaum möglich, jede erdenkliche Gefahr und das dadurch geforderte Verhalten zu normieren. Deshalb muss in Fällen, in denen zur Begründung einer Sorgfaltspflicht keine Normen vorhanden sind, auf den allgemeinen Gefahrensatz zurückgegriffen werden. Hierdurch verstärkt sich jedoch der Einfluss des Rückschaufehlers. Die Ermittlung der geforderten Sorgfaltspflicht anhand des Gefahrensatzes erfolgt nämlich in der Rückschau, sollte aber die Situation des Beschuldigten ex ante beurteilen. So ist z. B. in einem Fall das Aufstellen eines Grillgeräts die Schaffung einer Gefahr, welche die jederzeitige Beobachtung verlangt 41 und in einem anderen Fall wird gerade nicht verlangt, sich ununterbrochen ne-

Ist dies nicht möglich, so sind zusätzliche Massnahmen zu treffen, um einen Absturz zu verhindern.»

39 Urteil des Bundesgerichts 6B_543/2012 vom 11. April 2013 E. 1.4.2.

40 Rachlinski (Fn. 11), 608.

41 Urteil des Bundesgerichts 6S_529/2001 vom 18. Februar 2002 E. 2.b.bb. ben dem Grillgerät aufzuhalten. ${ }^{2}$ Eine Sorgfaltspflicht basierend auf dem Gefahrensatz kann sich somit je nach Fall verändern. Diese Flexibilität ist selbstverständlich gerade das Ziel des Gefahrensatzes, um auf die konkreten Situationen einzugehen. Dadurch entsteht aber auch eine erhöhte Gefahr bei der Beurteilung dem Rückschaufehler zu unterliegen.43 Muss aufgrund fehlender Vorschriften eine Sorgfaltspflicht in der Rückschau erstellt werden, sollte daher der Voraussehbarkeit eine erhöhte Aufmerksamkeit beigemessen werden. 44

\section{Die Voraussehbarkeit als eigen- ständiges Tatbestandsmerkmal}

24 Für die Definition der Voraussehbarkeit im Sinne der Sorgfaltspflichtverletzung behilft sich das Bundesgericht, ähnlich wie bei der adäquaten Kausalität, mit der Adäquanztheorie. Demzufolge ist die Gefahr des Erfolgseintritts für den Täter dann voraussehbar, wenn sein Verhalten geeignet ist, «nach dem gewöhnlichen Lauf der Dinge und den Erfahrungen des Lebens einen Erfolg wie den eingetretenen herbeizuführen oder mindestens zu begünstigen». 45

25 Aufgrund der Überschneidung respektive der Ähnlichkeit mit der Adäquanzprüfung kann für die Besprechung des Rückschaufehlers grundsätzlich auf das bei der Adäquanz Geschriebene verwiesen werden. Das Tatbestandsmerkmal der Voraussehbarkeit ist ebenfalls ex ante zu prüfen und unterliegt dadurch der Ge-

42 Urteil des Bundesgerichts 6S_728/2001 vom 6. März 2001 E. 3.

43 Schweizer (Fn. 1), 684 ff.

$44 \operatorname{Arzt}$ (Fn. 31), 306.

45 Anstatt vieler: BGE 135 IV 56 E. 2.1 S. 64. 
fahr eines Rückschaufehlers. 46

Dass das Bundesgericht die Formel der Adäquanz für die Beurteilung der Voraussehbarkeit der Sorgfaltspflichtverletzung heranzieht, ist m. E. nicht nachvollziehbar. 47 Schliesslich ist die Frage der adäquaten Kausalität aus Sicht eines sorgfältigen Dritten $\mathrm{zu}$ beantworten («allgemeine Lebenserfahrung»), die der Voraussehbarkeit dagegen aus Sicht des individuellen Beschuldigten («nach seinen persönlichen Verhältnissen»48). Die Vermischung der adäquaten Kausalität mit der Voraussehbarkeit schafft die Gefahr, dass die eigentliche Voraussehbarkeitsprüfung, die einem individualisierten Massstab folgt, in der objektiven Adäquanz untergeht.49 Vielleicht vermischt das Bundesgericht die beiden Aspekte bewusst aus der Not heraus, dass die individuellen Kenntnisse und Fähigkeiten des Beschuldigten aufgrund von Beweisschwierigkeiten nicht immer feststellbar sind.50 Eine solche Praxis verstösst jedoch m.E. gegen die Grundsätze ultra posse nemo tenetur51 und in dubio pro reo zugleich. So darf es nicht sein, dass bei einem Beschuldigten, bei

46 Frei (Fn. 19), 142.

47 Ohne überzeugende Begründung a.A. BGE 86 IV 153 E. 1 («Unerheblich ist auch der Einwand des Beschwerdeführers, er habe nicht voraussehen können, dass Kobel eine so gefährliche Ladung mit sich führe, und deshalb nicht mit dieser zusätzlichen Gefährdung rechnen müssen. $\mathrm{Ob}$ das Verhalten des Beschwerdeführers objektiv geeignet war, den Enderfolg herbeizuführen, hängt nicht davon $a b$, was der Beschwerdeführer persönlich voraussehen konnte. Die Frage, was der Täter voraussehen konnte oder musste, ist subjektiver Natur und beim Verschulden zu prüfen.»)

48 Art. 12 Abs. 3 Satz 2 StGB; vgl. BSK-StGB INiggli/Maeder (Fn. 20), Art. 12 N 100.

49 Vgl. Frei (Fn. 19), 97, 148.

5o Frei (Fn. 19), 157; BSK-StGB I-Niggli/Maeder (Fn. 20), Art. 12 N 103.

51 Über das Können hinaus wird niemand verpflichtet. dem die individuelle Fähigkeit nicht festgestellt werden kann, einfach (zu dessen Ungunsten) die Fähigkeit eines durchschnittlichen Dritten angenommen wird. $5^{2}$ Insbesondere unter der Berücksichtigung, dass das Gesetz ausdrücklich von den «persönlichen Verhältnissen» des Täters spricht.53

27 Es bleibt allerdings zu erwähnen, dass bei Anzeichen von verminderten Kenntnissen oder Fähigkeiten das Bundesgericht in früheren Entscheiden die objektive Zurechnung zwar bejahte, zugleich aber die individuelle Voraussehbarkeit verneinte. So wurde etwa im Fall BGE 122 IV 145 die adäquate Kausalität und somit die objektive Voraussehbarkeit durch das Bundesgericht bejaht. Aufgrund der Unerfahrenheit des Beschuldigten wurde aber die individuelle Voraussehbarkeit verneint und der Beschuldigte freigesprochen. 54

28 Entscheide wie dieser bestätigen, dass die Voraussehbarkeit nicht mit der adäquaten Kausalität gleichzusetzen ist. Auffällig ist, die Beurteilung der individuellen Fähigkeit wird vom Bundesgericht mit Hilfe objektiver Tatsachen vorgenommen.55 Ähnliches kennt man bei der Abgrenzung zwischen der bewussten Fahrlässigkeit und dem Eventualvorsatz. 56

\footnotetext{
Vgl. Frei (Fn. 19), 157.

Art. 12 Abs. 3 Satz 2 StGB.

BGE 122 IV 145 E. 3 S. 148.

BGE 122 IV 145 (zu wenig ausgebildeter Schlosser); 99 IV 127 (kein Expertenwissen bezüglich Gas); 99 IV 63 (Lehrling); 69 IV 228 (in intellektueller Hinsicht primitiver Eindruck).

56 BSK-StGB I-Niggli/Maeder (Fn. 20), Art. 12 N 103; BGE 130 IV 58 E. 8.4 S. 62.
} 

jektiver und subjektiver Voraussehbarkeit in der erwähnten Rechtsprechung des Bundesgerichts ist grundsätzlich lobenswert. Nur durch die klare Unterscheidung der objektiven Zurechnung und der individuellen Voraussehbarkeit ist eine wortgetreue Subsumtion der Fahrlässigkeit möglich. Das dogmatische Vorgehen in diesen Entscheiden zeigt aber eine Problematik auf, die auch heute noch in der Praxis des fahrlässigen Erfolgsdeliktes besteht: Die individuelle Voraussehbarkeit wird anhand objektiver Tatsachen «ausgeschlossen». Dies kommt bei näherer Betrachtung einer Beweislastumkehr gleich. Es wird gar nicht erst versucht, das Tatbestandsmerkmal der Voraussehbarkeit zu beweisen. Die Voraussehbarkeit wird aufgrund der bereits bejahten objektiven Zurechnung vermutet und dann anhand objektiver Tatsachen allenfalls verneint. 57

Bei der Beurteilung der subjektiven Voraussehbarkeit besteht somit die Gefahr, dass durch die Feststellung, ein Ereignis sei objektiv voraussehbar, auch automatisch angenommen wird, die konkrete Person hätte es tatsächlich vorausgesehen. Die Studien zum Rückschaufehler haben bisher nicht weiter untersucht, auf welcher Basis die Probanden dem Rückschaufehler unterlegen sind. Geschah dies aufgrund der Annahme, der Dritte hätte das Ereignis «vorausgesehen» (subjektiv) oder er «hätte es voraussehen müssen» (objektiv)? Die Erkenntnisse aus der Forschung weisen aber stark darauf hin, dass die Beurteilenden der betreffenden Person in der Rückschau zusätzliches Wissen unterstellen. Klare Be-

BGE 122 IV 145; 99 IV 127; 99 IV 63; 69 IV 228. lege dazu gibt es bisher jedoch nicht. $5^{8}$ Deshalb kann nicht abschliessend festgestellt werden, ob zwischen der objektiven Voraussehbarkeit (bei der adäquaten Kausalität) und der subjektiven Voraussehbarkeit (bei der Sorgfaltspflichtverletzung) ein Unterschied hinsichtlich des Rückschaufehlers besteht.

\section{Sportschützen-Fall}

31 Das Bundesgericht hatte sich zwei Mal mit dem sogenannten Sportschützen-Fall zu befassen.59 Bei der zweiten Beurteilung vor Bundesgericht wurde die Beschwerde des Beschuldigten X aufgrund eines Rückschaufehlers gutgeheissen 60 und es erfolgte daraufhin vor Obergericht ein Freispruch in dubio pro reo. ${ }^{61}$

32 Der Beschuldigte X hat als praktizierender Arzt und Psychoanalytiker eine Unbedenklichkeits-erklärung zugunsten der Sportschützin A ausgestellt. Dadurch bekam diese ihre bei der Polizei sichergestellte Waffe zurück, mit welcher sie kurze Zeit später einen Mann anschoss. Der durch das Gericht beauftragte Sachverständige musste ex post beurteilen, wie eine sorgfältige Fachperson in der konkreten Situation ex ante geurteilt hätte. Dabei wusste der Sachverständige vom Ausgang, vom zweiten Zwischenfall mit der Waffe und von der daraus folgenden Körperverletzung. Dem Sachverständigen wurden Akten vorgelegt, die ihm ein nachträgliches Wissen vermittelten, dass der Beschuldigte in seiner Beurteilung

$5_{8}$ Rachlinski (Fn. 11), 592.

59 BGE 135 IV 56; Urteil des Bundesgerichts 6B_365/2010 vom 14. März 2011.

60 Urteil des Bundesgerichts 6B_365/2010 vom 14. März 2011 E. 5.

61 Urteil des Obergerichts ZH SB110210 vom 8. September 2014 E. 6.h. 
zur Tatzeit unmöglich haben konnte. In seinem Entscheid äusserte sich das Bundesgericht denn auch erstmals explizit zum Problem des Rückschaufehlers. Weil im Gutachten der Wissensunterschied nicht genügend berücksichtigt wurde, hiess das Bundesgericht die Beschwerde gut und kassierte den Schuldspruch des Obergerichts. ${ }^{62}$ Weiter forderte es das Obergericht auf, ein neues Gutachten zu erstellen und - falls es darin nicht mehr möglich sei, die Relevanz des sorgfaltswidrigen Verhaltens festzustellen - den Beschuldigten in dubio pro reo freizusprechen. ${ }^{63}$ Das Obergericht sprach den Beschuldigten daraufhin frei. 64

33 Diese Ausgangslage gleicht einer Studie zum Rückschaufehler von LaBine/LaBine. ${ }^{65}$ In ihrem Experiment ging es ebenfalls darum, die Haftung eines Therapeuten zu beurteilen, der die Gefahr eines Gewaltausbruches durch seinen Patienten als unbedenklich beurteilte. Dabei zeigte sich eine klare Überschätzung der Voraussehbarkeit, wenn bekannt war, dass der Patient des Therapeuten später tatsächlich gewalttätig wurde. 66

34 In seinem Präjudiz erkannte das Bundesgericht erstmals, dass die ex ante Beurteilung aufgrund des Rückschaufehlers $\mathrm{zu}$ falschen Urteilen führen kann. Im konkreten Fall hält das Bundesgericht in seinen Erwägungen unter anderem fest: «[...] der Experte [hätte] festhalten müs-

62 Urteil des Bundesgerichts 6B_365/2010 vom 14. März 2011 E. 4.13.

63 Urteil des Bundesgerichts 6B_365/2010 vom 14. März 2011 E. 5.

64 Urteil des Obergerichts ZH SB110210 vom 8. September 2014 E. 6.h.

65 LaBine/LaBine (Fn. 5), $501 \mathrm{ff}$.

66 LaBine/LaBine (Fn. 5), 510. sen, welchen Wissensstand eine sorgfältige Fachperson im Tatzeitpunkt erlangt hätte. Dies ergibt sich aus dem Gutachten nicht.»67 Im nun bereits dritten Prozess vor Obergericht stellte der Gutachter wiederum fest, dass im Tatzeitpunkt eine Gefahr für Dritte voraussehbar war. Weil sich seine Ausführungen aber lediglich auf einen forensisch ausgebildeten Psychiater bezogen, was der Beschuldigte nicht ist, konnten die Richter nicht zweifelsfrei feststellen, ob der Beschuldigte persönlich die Drittgefährdung voraussehen konnte. ${ }^{68}$ Folglich sprach das Obergericht den Beschuldigten in dubio pro reo frei.

\section{Methoden zur Verminderung des Rückschaufehlers im Strafrecht}

35 Die bisher einzige als wirksam anerkannte Methode zur Verminderung des Rückschaufehlers erfolgt, wie bereits erwähnt, durch die bewusste Auseinandersetzung mit den alternativen Ausgangsmöglichkeiten. In Experimenten wurde dies erreicht, indem der Beurteilende zu jedem möglichen Ausgang zusätzlich ein oder mehrere Argumente liefern musste. ${ }^{69}$ Die praktische Umsetzung dieser Methode in der richterlichen Beurteilung scheint mir jedoch schwierig bis unmöglich: Die möglichen Varianten von Ausgangsmöglichkeiten, die ein Richter berücksichtigen muss, sind um einiges umfangreicher als dies in Experimenten dargestellt werden kann. Es steht dem Richter nicht einfach eine beschränkte Auswahl von möglichen Folgen eines Verhaltens zur Verfügung, von der er dann für jede Variante

67 Urteil des Bundesgerichts 6B_365/2010 vom 14. März 2011 E. 4.13.1.

68 Urteil des Obergerichts ZH SB110210 vom 8. September 2014 E. 6.g.

69 Arkes et al. (Fn. 13), 305. 
Argumente niederschreiben kann.

36 Die möglichen Ansätze, den Rückschaufehler im Rechtssystem zu vermeiden, sind damit aber noch nicht ausgeschöpft. In der Folge werden die zwei meiner Ansicht nach besten Debiasing-Methoden dargelegt. Der Rückgriff auf ex ante Hinweise (1) und die strikte Beachtung der Prozessgrundsätze (2). Als Drittes wird die in der Rechtsliteratur häufig genannte Debiasing-Methode der Zweiteilung des Prozesses (Bifurcation70) untersucht. Weil diese bisher immer nur bei Zivilrechtsfällen zur Anwendung kam, wird hier ihre Tauglichkeit für das Strafrecht überprüft (3).

\section{Rückgriff auf ex ante Hinweise}

37 Die Berücksichtigung von Hinweisen, die bereits zum Tatzeitpunkt bestanden, geben dem Beobachter einen realistischen Einblick in die damalige Auffassung, welche Gefahr ein konkretes Verhalten birgt.71 Als Beispiel sei der bereits angesprochene Art. 46 Abs. 2 BauAV noch einmal erwähnt. Würde diese Norm nicht bestehen, müsste sich das Gericht bei einem Unfall mit der Frage auseinandersetzen, ab welchem Abstand der Beschuldigte die Gefahr eines Absturzes hätte voraussehen müssen. Dabei würde diese Beurteilung nach den bisherigen Erkenntnissen durch den Rückschaufehler beeinflusst werden. Auch unter Einhaltung der $30 \mathrm{~cm}$ könnte demnach dem Beschuldigten aufgrund des eingetrete-

70 Pengelley (Fn. 11), 70 f.; Smith/Greene (Fn. 11), $508 \mathrm{f}$; Wexler/Schopp, How and When to Correct for Juror Hindsight Bias in Mental Health Malpractice Litigation: Some Preliminary Observations, in: Behavioral Sciences \& the Law 7/1989, 485, 493 f.

71 Guthrie/Rachlinski/Wistrich (Fn. 13), 825, 828 f.; Rachlinski (Fn. 11), 608; Schweizer (Fn. 1), $676 \mathrm{ff}$. nen Erfolgs ein schuldhaftes Verhalten vorgeworfen werden. Mit der angesprochenen Norm wurde jedoch ein objektiver Bezugspunkt vor dem Tatzeitpunkt geschaffen. Der rückschauende Beobachter braucht sich keine Gedanken mehr über den objektiv geforderten Abstand zu machen.

38 Folglich kann der Rückschaufehler durch die Beachtung von ex ante Normen vermindert, wenn nicht sogar gänzlich verhindert werden. ${ }^{2}$ Je konkreter eine Regel ausformuliert ist, desto weniger Spielraum bleibt dabei für die Rückschauverzerrung.

39 Das Heranziehen von ex ante Hinweisen sollte m. E. nicht auf die Rechtsquellen beschränkt bleiben, die jeweils beim Tatbestandsmerkmal der Sorgfaltspflicht genannt werden.73 Nebst bestehenden Gesetzen, Vorschriften, Reglementen oder Merkblättern können nämlich auch zum Tatzeitpunkt bestehende Prognosen, Gutachten, Aussagen oder beschränkt sogar Gewohnheitsrecht74 als Massstab für die Zumutbarkeit herangezogen werden. Bei einem Lawinenunglück durch eine geführte Skitourengruppe kann im Strafprozess gegen den Tourenführer das Lawinenbulletin vom Unfalltag einen

72 Guthrie/Rachlinski/Wistrich (Fn. 13), 828; Peter Jäger/Angela Schweiter, Der Hindsight Bias (Rückschaufehler) - ein grundsätzliches Problem bei der Beurteilung ärztlichen Handelns in Arzthaftpflichtund Arztstrafprozessen, in: Schweizerische Ärztezeitung 86/2005, 1940, 1942; Rachlinski (Fn. 11), 608 ff.; Schweizer (Fn. 1), 676 ff.

73 Vgl. BGE 140 II 7 E. 3.4 S. 10; 130 IV 7 E. 3.3 S. 11; Urteil des Bundesgerichts 6B_543/2012 vom 11. April 2013 E. 1.3.2;Donatsch/Tag (Fn. 19), 347 ff.; BSK-StGB I-Niggli/Maeder (Fn. 20), Art. 12 N 111; Schweizer (Fn. 1), 676 ff.; Seelmann (Fn. 36), 168; Trechsel/Noll (Fn. 20), 270 ff.

74 Pengelley (Fn. 11), 64; Rachlinski (Fn. 11), 608 ff. 
wichtigen ex ante Hinweis darstellen.75 Das Bundesgericht hat bereits von derartigen ex ante Hinweisen Gebrauch gemacht: In einem Entscheid verneinte es die Erkennbarkeit einer Lawine nicht zuletzt wegen des Lawinenbulletins und den Aussagen Einheimischer. ${ }^{76}$

40 Auch wenn konkrete oder klar formulierte ex ante Hinweise bestehen, bleibt jedoch das Problem, dass diese meist nur einen objektiven Einblick geben können. Bleibt man beim Beispiel der geführten Skitour, wäre z.B. denkbar, dass der Tourenführer beim Aufstieg Anzeichen für eine erhöhte lokale Lawinengefahr bemerkte und diese mit dem Gedanken missachtete: «Es wird schon nichts passieren.» Die Gefahr war nun für den konkreten Beschuldigten erkennbar und sein Verhalten, die Tour trotzdem durchzuführen, wäre somit fahrlässig. Bei strikter Berücksichtigung von ex ante Hinweisen würde der Beschuldigte aufgrund des Lawinenbulletins freigesprochen. Eine gesetzliche Fixierung an ex ante Hinweise kann somit nicht die Lösung des Problems sein. Weiter gilt es bei solchen nichtstaatlichen Sorgfaltspflichten die Urheber zu berücksichtigen. Je nach Urheber werden unterschiedliche Interessen vertreten, welche dann zu einer einseitigen Beurteilung führen können. 77 Die Berücksichtigung von ex ante Hinweisen verhindert aber immerhin den Rückgriff auf die für Verzerrungen anfällige Generalklausel. Die Gerichte bleiben mit oder ohne ex ante Hinweise extrem gefordert in ihrer rückschauenden Beurteilung.

\footnotetext{
75 Schweizer(Fn. 1), 676.

76 BGE 116 IV 182 E. 5 S. 186.

77 Schweizer (Fn. 1), 682 f.
}

\section{Beweiswürdigung im Strafprozess}

41 Strafprozessuale Grundsätze können kognitive Täuschungen minimieren.78 Tatsache ist, dass der Rückschaufehler insbesondere in Zweifelsfällen ausschlaggebend sein kann.79 Gemäss der Unschuldsvermutung im Strafrecht müsste in solchen Fällen zugunsten des Beschuldigten immer auf Freispruch erkannt werden. ${ }^{80}$ Der Richter muss auf der Suche nach der materiellen Wahrheit vom Sachverhalt und der Schuld des Beschuldigten überzeugt sein und darf keine Zweifel mehr haben. ${ }^{81}$

42 Der strafrechtliche Grundsatz der Unschuldsvermutung scheint auf den ersten Blick ein taugliches Instrument zur Verminderung der verzerrten Rückschau zu sein. Je strenger die Beweiswürdigung, umso weniger Platz bleibt für kognitive Fehler. ${ }^{82}$ Dennoch bleibt in der menschlichen Entscheidungsfindung Raum für kognitive wie auch emotionale Einflüsse. ${ }^{8}$ Das Problem besteht darin, dass kognitive Fehler Einfluss nehmen, bevor überhaupt Zweifel aufkommen. Durch den Rückschaufehler wird die Geschichte stichhaltig und von Zweifeln befreit. Bestehen keine Zweifel beim Richter, wird die Unschuldsvermutung wohl gar nicht in Betracht gezogen.84 Der Rückschau-

78 Sommer (Fn. 35), 176

79 Pengelley (Fn. 11), 57; Rachlinski (Fn. 11), 588 ff.

80 Andeas Donatsch/Christian Schwarzenegger/Wolfgang Wohlers, Strafprozessrecht, 2. Aufl., Zürich 2014, 58 ff.; Niklaus Oberholzer, Grundzüge des Strafprozessrechts, 3. Aufl., Bern 2012, 688 ff.

81 Donatsch/Schwarzenegger/Wohlers (Fn. 80), 58 f.; Roger Groner, Beweisrecht, Beweise und Beweisverfahren im Zivil- und Strafrecht, Bern 2011, 180 f.; Oberholzer (Fn. 80), 691 f.

82 Rachlinski (Fn. 11), 606 f.

83 Stephan Bernard, In dubio pro reo?, in: forumpoenale 2013, 112, 114 f.; Sommer (Fn. 35), 161 ff.

84 Vgl. Guthrie/Rachlinski/Wistrich (Fn. 13), 820. 
fehler kommt dem Grundsatz der Unschuldsvermutung zuvor. Das hat nicht zuletzt der bereits erwähnte Sportschützen-Fall deutlich aufgezeigt.

\section{Zweiteilung des Prozesses}

43 Die wohl meistgenannte DebiasingMethode in der angloamerikanischen Rechtsliteratur zum Rückschaufehler ist die Zweiteilung des Prozesses (Bifurcation). ${ }^{85}$ Dabei wird über die Tatbestandsmerkmale die in der Rückschau beurteilt wird in einem eigenen ersten Teilprozess entschieden. Im angloamerikanischen Zivilprozess kann so zuerst über die Sorgfaltspflichtverletzung entschieden werden, ohne dass der Richter respektive die Geschworenen vom daraus resultierenden Schaden Kenntnis haben. Der Schaden wird erst in einem zweiten Teilprozess bekannt, in welchem dann der Schadenersatz gesprochen wird. Das Ausmass des Schadens hat damit keinen Einfluss mehr auf die Beurteilung der Sorgfaltspflichtverletzung und der Rückschaufehler wird nachweislich vermindert. 86

44 Mit Art. 342 Abs. 1 StPO besteht im Schweizer Strafprozess ebenfalls die Möglichkeit der Zweiteilung des Verfahrens. Es stellt sich jedoch heraus, dass sowohl das Schuld- als auch das Tatinterlokut nicht geeignet sind, um den Rückschaufehler zu umgehen. Erforderlich ist nicht die Abgrenzung der Schuldfrage, wie dies aktuell möglich wäre. Für ein erfolgreiches Debiasing müsste das Tatbestandsmerkmal des Erfolgs getrennt beurteilt werden können (Erfolgsinterlokut). Das würde bedeuten, dass im ersten Teilprozess nur die Sorgfaltspflicht-

85 Pengelley (Fn. 11), 70 f.; Smith/Greene (Fn. 11), 508 f.; Wexler/Schopp (Fn. 70), 493 f.

86 Smith/Greene (Fn. 11), $514 \mathrm{ff}$. verletzung behandelt und der Erfolg verschwiegen wird. Auf den Sportschützenfall bezogen heisst das, dass das Gericht sowie der beauftragte Gutachter im ersten Teilprozess nur beurteilen muss, ob der Beschuldigte bei seiner Diagnose und der Ausstellung der Unbedenklichkeitserklärung die geforderte fachliche Sorgfalt eingehalten hat. Erst im zweiten Teilprozess würde dann der tatbestandsmässige Erfolg und die natürlichen Kausalität geprüft sowie das Strafmass bestimmt werden.

45 Die Prüfung der Sorgfaltspflichtverletzung könnte mit der oben geschilderten Zweiteilung allenfalls ohne die Gefahr eines Rückschaufehlers beurteilt werden. Schwieriger wird dies jedoch bei der Beurteilung der adäquaten Kausalität sowie der Voraussehbarkeit des Erfolgs. Gemäss aktueller Rechtsprechung muss der adäquate Kausalzusammenhang zwischen der Ursache und dem «Erfolg in der Art des eingetreten» hergestellt werden. Dasselbe gilt bei der Prüfung der Voraussehbarkeit. Es muss der konkret eingetretene Erfolg adäquat kausal und voraussehbar gewesen sein. Ohne Kenntnis über den eingetretenen Erfolg ist die Prüfung der beiden Tatbestandsmerkmale somit nicht möglich.

46 Hinzu kommt die Problematik, dass dem Richter alleine durch das Bestehen des Prozesses schon klar ist, dass ein tatbestandsmässiger Erfolg eingetreten sein muss. ${ }^{87}$ Gänzlich unmöglich ist die Verschleierung des Erfolgs in den durch Medien bekannten Fällen. Die Methode der Zweiteilung zur Behebung des Rückschaufehlers scheint mir daher für das Strafrecht ungeeignet.

87 Wexler/Schopp (Fn. 70), 494. 


\section{Fazit}

47 Die kognitiven Täuschungen im Entscheidprozess des Menschen beschäftigen die Rechtswissenschaft zunehmend. Zahlreiche Studien haben inzwischen die Existenz des Rückschaufehlers untersucht und mit Experimenten nachgewiesen. Dennoch scheint der Rückschaufehler, was die Beurteilung vor Gericht angeht, kaum greifbar. Reale Gerichtsentscheide lassen sich im Nachhinein nur schwerlich auf eine mögliche Rückschauverzerrung hin untersuchen. Hinzu kommt die Tatsache, dass jeder Mensch Gefahr läuft, dem Rückschaufehler zu verfallen und ein Urteil daher als richtig erscheinen kann, obwohl es durch den Rückschaufehler verfälscht wurde. Rachlinski meint sogar, dass ein Einzelfall, der vom Rückschaufehler betroffen ist, fairer erscheint, als wenn er durch Debiasing korrigiert würde. Dies aus dem einfachen Grund, weil generell jeder Betrachter, der den Erfolg kennt, das Gefühl haben wird, seine in Wahrheit verfälschte rückschauende Beurteilung sei korrekt. 88

Dass der Rückschaufehler unsere Entscheidungen beeinflussen kann, steht ausser Zweifel. Inwiefern er tatsächlich das Schweizerische Strafrecht beeinflusst und sich auf das Urteil und den Beschuldigten auswirkt, lässt sich aber nur schwer beantworten. Die Auseinandersetzung mit dem Fahrlässigkeitsdelikt hat die Bereiche aufgezeigt, in denen die Gefahr eines Rückschaufehlers am grössten ist. Bei der Frage nach der Zurechnung (adäquate Kausalität), der Sorgfaltspflicht und der Voraussehbarkeit kann der Rückschaufehler ein Urteil massgeblich beeinflussen.
49 Die Auswirkungen auf das System insgesamt mögen gering sein. Die verschiedenen Fälle sind unterschiedlich empfänglich für kognitive Verzerrungen und der Einfluss des Rückschaufehlers muss daher nicht zwingend jedes Urteil beeinflussen. ${ }^{89}$ Für den einzelnen Beschuldigten in einem Strafprozess, der vom Rückschaufehler beeinflusst wird, kann der Effekt jedoch von entscheidender Bedeutung sein. Bestätigt wird diese Befürchtung durch den erwähnten Sportschützen-Fall.9o

5o Im vorliegenden Beitrag habe ich Methoden aufgezeigt, die geeignet sein können den Rückschaufehler bestenfalls zu minimieren. Die wirkungsvollste Lösung erscheint mir die Beachtung von ex ante Hinweisen, soweit solche bestehen. Umgekehrt bedeutet dies, dass im Nachhinein bekannte Tatsachen eher zurückhaltend berücksichtigt werden sollten. Dieses Vorgehen zu normieren oder eine Beweisregel einzuführen, erscheint jedoch nicht angebracht. Dafür reichen die aktuellen Grundsätze im Strafprozessrecht aus. Insbesondere dem Grundsatz in dubio pro reo sollte aber in diesem Bereich besondere Aufmerksamkeit geschenkt werden. Die Methode der Zweiteilung ist für das Strafrecht in der Schweiz hingegen nicht durchführbar.

51 Die Entwicklung der Rechtsprechung, die adäquate Kausalität immer mehr auszudehnen, wurde in der Lehre bereits mehrfach kritisiert.91 Leider lässt sich

89 Pengelley (Fn. 11), 57; Rachlinski (Fn. 11), 588 ff.

90 Urteil des Bundesgerichts 6B_365/2010 vom 14. März 2011.

$91 \operatorname{Arzt}$ (Fn. 31), 305 f.; Frei (Fn. 19), 118 ff., 612 ff.; Killias et al. (Fn. 20), 407; BSK-StGB I-Niggli/ Maeder (Fn. 20), Art. 12 N 95; Trechsel/Noll (Fn. 20), 270. 
nicht nachweisen, ob und wie stark der Rückschaufehler an dieser Entwicklung mitschuldig ist. Einzelne Stimmen in der deutschen Rechtswissenschaft gehen allerdings von einem klaren Zusammenhang aus. ${ }^{22}$ Die vermehrte Auseinandersetzung mit der adäquaten Kausalität und deren Alternativen ist unumgänglich. Mit dem Rückschaufehler zeigt sich nämlich eine weitere Schwäche der aktuellen Adäquanztheorie.

52 Abschliessend kann festgehalten werden, dass die Schweizer Rechtsprechung mit dem erstmaligen Anerkennen des Rückschaufehlers einen ersten Schritt hin zur Berücksichtigung kognitiver Täuschungen gemacht hat. Es bleibt zu hoffen, dass sich die Gerichte in Zukunft vermehrt mit der Rückschauproblematik auseinandersetzen. Einen dringenden Handlungsbedarf auf Seiten des Gesetzgebers gibt es meiner Auffassung nach aber nicht, zumal das gegenwärtige Wissen noch nicht ausreicht, um wirksame Anpassungen am Gesetz vorzunehmen. Bis zu einem gewissen Grad muss man sich gegenwärtig mit Second-BestLösungen 93 zufrieden geben.

92 Falk(Fn. 3), 10 ff.; Sommer (Fn. 35), 171 f.

93 Rachlinski (Fn. 11), $624 \mathrm{f}$. 